\title{
Prevalence and Risk Factors associated with Extended Spectrum Beta Lactamase Producing Escherichia coli and Klebsiella pneumoniae Isolates in Hospitalized Patients in Kashan (Iran)
}

\author{
Mohammad Reza Sharif ${ }^{1}$, Babak Soltani $^{1}$, Alireza Moravveji ${ }^{2}$, Mahzad Erami $^{3}$, Nika Soltani $^{4}$
}

${ }^{1}$ Department of Pediatrics, Kashan University of Medical Sciences, Kashan, Iran

${ }^{2}$ Department of Community Medicine, Trauma Research Center, Kashan University of Medical Sciences, Kashan, Iran

${ }^{3}$ Department of Microbiology, Kashan University of Medical Sciences, Kashan, Iran

${ }^{4}$ Student Research Committee, Tehran University of Medical Sciences, Tehran, Iran

\section{Type of article: Original}

\begin{abstract}
Introduction: Production of extended spectrum beta lactamase (ESBL) is an important mechanism of antimicrobial resistance in Escherichia coli (E. coli) and Klebsiella pneumoniae (K. pneumoniae) isolates. This study was performed to determine the prevalence and risk factors associated with ESBL producing strains of $E$. coli and K. pneumoniae.

Methods: In this cross-sectional study, 250 strains (134 E. coli and $116 \mathrm{~K}$. pneumoniae) were obtained, and ESBL producing isolates were detected by the combination disk test in Shahid Beheshti Hospital in Kashan, Iran, from February 2012 to June 2013. Antimicrobial resistance was screened by the disk diffusion method and was confirmed by E-test. Furthermore, risk factors of ESBL producing E. coli and $K$. pneumoniae microorganisms were determined. Data were analyzed by SPSS version 16, using descriptive statistics, chi-squared, independentsamples t-test, and logistic regression analysis.

Results: One hundred and two (40.8\%) of all strains were ESBL producers, of which 54 (52.9\%) were E. coli and $48(47.1 \%)$ were $K$. pneumoniae $(\mathrm{p}=0.86)$. Furthermore, $40.3 \%$ of $E$. coli and $41.4 \%$ of $K$. pneumoniae isolates were ESBL producers $(\mathrm{p}=0.86)$. The most antimicrobial resistance was to ampicillin, and no imipenem resistance was detected. Risk factors for ESBL producing $E$. coli included admission duration exceeding 7 days $(\mathrm{p}=0.011)$ and antibiotic use in the last month $(\mathrm{p}<0.001)$, and the associated risk factor for ESBL producing $K$. pneumoniae was antibiotic use during the recent month $(\mathrm{p}=0.002)$.

Conclusion: This study identified a relatively high prevalence of ESBL production among $E$. coli and $K$. pneumoniae strains. Furthermore, anti-bimicrobial use and admission duration were risk factors for ESBL producing isolates. Therefore, more comprehensive investigations are needed for the development of new strategies to control the dissemination of these microbes.
\end{abstract}

Keywords: Escherichia coli, Klebsiella pneumoniae, ESBL, risk factors

\section{Introduction}

The emergence of extended spectrum beta lactamase (ESBL) among gram negative bacteria has become a major public health problem during recent years (1). It confers a substantial resistance to penicillins, and a broad spectrum of cephalosporins and monobactams, but not to carbapenems. Plasmids often are the major cause of ESBL producing organisms, which occasionally are transmitted between different strains of enteric gram negative rods (2). E. coli and K. pneumoniae have been indicated as the most common causes of ESBL producing bacteria (3). Today, ESBL producers have been recognized as the mainstay causes of hospital and community-acquired infections globally $(1,3)$. Isolates that express ESBL phenotypes and hydrolize the beta lactam antibiotics are often multi-drug resistant (MDR) (4). Carbapenems have been recommended as the choice of therapy for serious infections caused by ESBL producers (5). However, the increased use of carbapenems has led to emerging carbapenem resistance by the

\section{Corresponding author:}

Babak Soltani, Department of Pediatrics, Kashan University of Medical Sciences, Kashan, Iran.

Tel: +98.3155575840, Fax: +98.3155548900, E-mail: babak_soltani1969@yahoo.com

Received: November 11, 2015, Accepted: January 24, 2016, Published: March 2016

iThenticate screening: January 24, 2016, English editing: February 28, 2016, Quality control: March 04, 2016

(C) 2016 The Authors. This is an open access article under the terms of the Creative Commons Attribution-NonCommercialNoDerivs License, which permits use and distribution in any medium, provided the original work is properly cited, the use is non-commercial and no modifications or adaptations are made. 
creation of carbapenemase among gram negative bacteria (6). The prevalence of ESBL producing strains varies in different parts of the world, with lower rates (3-8\%) in Singapore, Japan, and Sweden despite the higher rates in Italy $(37 \%)$, Turkey $(58 \%)$, and Iran $(59.2 \%)(7,8)$. Physicians should be vigilant against the spread of these infections and their bacterial resistance. In this investigation, we determined the prevalence and antibiotic resistance patterns of ESBL positive isolates, including E. coli and $K$. pneumoniae, and their associated risk factors among patients admitted to hospitals in Kashan, Iran.

\section{Material and Methods}

\subsection{Study design and participants}

In this cross-sectional study, which was conducted from February 2012 to June 2013 at Shahid Beheshti Hospital of Kashan, 250 isolates (E. coli and $K$. pneumoniae) from clinical specimens were obtained. The patients who were admitted to the ICU, internal medicine, pediatric, infectious, surgery, and gynecology wards were selected nonrandomly, and their laboratory tests were conducted in the microbiology laboratory at the hospital. After obtaining informed consent from the patients with positive cultures, their data were written in questionnaires. The data included age, gender, clinical specimen, admission ward, medical disorder history, history of antibiotic or steroid use during the past month, and history of hospitalization in the last three months. Sample size was estimated based on the results of a similar previous study with ESBL prevalence of $15.4 \%$, a confidence interval of $95 \%$, and $d=0.1$ (9).

\subsection{Research Ethics}

The study was approved by The Ethics Committee at Kashan University of Medical Sciences (approval number 2736). The patients were not charged, and their information was kept confidential.

\subsection{Collection of samples and bacterial identification}

All clinical samples (urine, stool, blood, peritoneal, and pleural fluids) were cultured on blood and MacConkey agar plates (Merck, Germany) at $37{ }^{\circ} \mathrm{C}$ for 24 hours, and the yielded organisms were identified according to colonial morphologies, biochemical reactions, and gram staining. On the blood agar culture, colonies of $E$. coli were round in shape with a flat surface, grayish color, and juicy consistency with diameters of 1-2 mm, but K. pneumoniae were dome shaped, mucoid, large colonies with a coalescence tendency. On the MacConkey culture, E. coli colonies were red, whereas $K$. pneumoniae colonies were large mucoid dark pink (10). The biochemical characteristics of $E$. coli included positive Methyl Red (MR), indole production, negative Simmon citrate, negative Voges-Proskauer (VP), negative urease, and motile, but the biochemical specifications of $K$. pneumoniae were positive urease, no indole production, positive VP, positive Simmon citrate, variable MR, and non-motile. Microscopy alone cannot be used to discriminate between the two organisms (10).

\subsection{Antimicrobial Resistance}

According to the Clinical and Laboratory Standards Institute's (CLSI's) guidelines, the antibiotic resistance of isolates was evaluated (11). E. coli and K. pneumoniae colonies were inoculated onto Mueller-Hinton agar (Merck, Germany) and by the Kirby-Bauer disk diffusion method (Mast, UK), screening for antibiotic resistance was done. The following disks were put on Mueller-Hinton agar and incubated for 24 hours at $37{ }^{\circ} \mathrm{C}$, i.e., $30 \mu \mathrm{g}$ amikacin, 10 $\mu \mathrm{g}$ ampicillin, $20 / 10 \mu \mathrm{g}$ amoxicillin-clavulanic acid, $30 \mu \mathrm{g}$ ceftriaxone, $30 \mu \mathrm{g}$ ceftazidime, $5 \mu \mathrm{g}$ ciprofloxacin, $10 \mu \mathrm{g}$ gentamycin and $10 \mu \mathrm{g}$ imipenem. Inhibition zones of $\leq 13 \mathrm{~mm}$ around ampicillin, amoxicillin-clavulanic acid, ceftriaxone and imipenem, $\leq 12 \mathrm{~mm}$ around gentamycin, $\leq 14 \mathrm{~mm}$ around ceftazidime and amikacin and $\leq 15 \mathrm{~mm}$ around ciprofloxacin were considered resistant (11). The minimal inhibitory concentration (MIC) that was calculated by the Epsilometer test (E-test) (Liofilchem, Italy) was used as the confirmatory test of anti-microbial resistance. MICs $\geq 32 \mu \mathrm{g} / \mathrm{ml}$ for amikacin and ampicillin, $\geq 64 \mu \mathrm{g} / \mathrm{ml}$ for ceftriaxone, $\geq 32 / 16 \mu \mathrm{g} / \mathrm{ml}$ for amoxicillin-clavulanic acid, $\geq 8 \mu \mathrm{g} / \mathrm{ml}$ for gentamycin, $\geq 32 \mu \mathrm{g} / \mathrm{ml}$ for ceftazidime and $\geq 16 \mu \mathrm{g} / \mathrm{ml}$ for imipenem were considered resistant. MDR was defined as resistance to $\geq 3$ antibiotics (11). Detection of antimicrobial resistance was performed by only one person in the laboratory.

\subsection{Detection of ESBL strains}

The ESBL screening method on Mueller-Hinton agar was conducted by disk diffusion test using $30 \mu \mathrm{g}$ ceftazidime and $30 \mu \mathrm{g}$ cefotaxime disks (Mast, UK). The inhibition zones of $\leq 22 \mathrm{~mm}$ around ceftazidime and $\leq 27 \mathrm{~mm}$ around cefotaxime were considered resistant (11). Phenotypic confirmation of ESBL production was performed by combined disk test (Mast, UK) using cefotaxime $(30 \mu \mathrm{g})$ and ceftazidime $(30 \mu \mathrm{g})$ disks alone and with clavulanic 
acid $(10 \mu \mathrm{g})$. An increase of $\geq 5 \mathrm{~mm}$ in the zone of inhibition around any anti-microbial agent in combination with clavulanic acid versus its zone alone was considered ESBL production (11). Positive and negative control isolates included E. coli ATCC 25922 and K. pneumoniae ATCC 7006039 (Mast, UK) (11). ESBL screening and its confirmation were conducted only by one person in the laboratory.

\subsection{Statistical analysis}

The data were entered into SPSS software version 16 (SPSS, Inc. Chicago, Illinois, USA). Descriptive results were evaluated by their frequencies, means, and standard deviations. Normal distribution of continuous data was detected by the Kolmogorov-Smirnov test. Comparison of the mean age of patients between the two groups was done by the independent-samples t-test. Demographic data, characteristics of cases, and antibiotic resistance patterns were estimated by the chi-squared test. To analyze the association of risk factors with ESBL production, the two-step logistic regression test was used. In the first step, the variables that were unlikely to be associated with ESBL strains ( $p>0.2$ in univariate logistic regression) were excluded from the final analysis. In the second step, any eligible factors were entered in multivariate logistic regression analysis and two-tailed $\mathrm{p}<0.05$ was considered statistically significant.

\section{Results}

From the 250 isolates that were produced from samples, including urine $(\mathrm{n}=134)$, stool $(\mathrm{n}=76)$, blood $(\mathrm{n}=16)$, pleural fluid $(\mathrm{n}=12)$, and ascitic fluid $(\mathrm{n}=12), 134(53.6 \%)$ were E. coli and $116(46.4 \%)$ were K. pneumoniae. The age range of cases was 2-81 with a mean of $34.9 \pm 24.5$. One hundred and thirteen of the participants were females $(45.2 \%)$, and $137(54.8 \%)$ were males. One hundred and two $(40.8 \%)$ of all of the strains were ESBL producers, of which $54(52.9 \%)$ were $E$. coli and $48(47.1 \%)$ were $K$. pneumoniae $(\mathrm{p}=0.86)$. Moreover, $40.3 \%$ of $E$. coli and $41.4 \%$ of $K$. Pneumoniae isolates were ESBL producing pathogens $(\mathrm{p}=0.86)$. There was no significant association between clinical samples and the production of ESBL $(\mathrm{p}=0.88)$. Moreover, no association was detected between admission wards and ESBL producers $(\mathrm{p}=0.053)$. Of the ESBL producing strains, $58.8 \%$ were males, and $52 \%$ of the non-ESBL producing strains were males $(\mathrm{p}=0.29)$. The mean ages of the ESBL positive and ESBL negative cases were $38.1 \pm 25.8$ and $32.6 \pm 23.5$, respectively $(\mathrm{p}=0.08)$. Antibiotic resistance patterns of the isolates are provided in Tables 1 and 2.

Table 1. Antibiotic resistance rates (\%) of extended spectrum beta lactamase (ESBL)-positive and ESBL-negative Escherichia coli strains

\begin{tabular}{|l|l|l|l|l|}
\hline Antibiotics & ESBL-positive $(\mathrm{n}=54)$ & ESBL-negative $(\mathrm{n}=80)$ & Total $(\mathrm{n}=134)$ & $\mathrm{p}^{\mathrm{a}}$ \\
\hline Amikacin & 48.1 & 12.5 & 26.9 & $<0.001$ \\
\hline Ampicillin & 90.7 & 66.2 & 76.1 & 0.001 \\
\hline Ceftazidime & 37 & 26.2 & 30.6 & 0.2 \\
\hline Ceftriaxone & 38.9 & 18.8 & 26.9 & 0.01 \\
\hline Ciprofloxacin & 38.9 & 10 & 21.6 & $<0.001$ \\
\hline Co-Amoxiclav & 38.9 & 18.8 & 26.9 & 0.01 \\
\hline Gentamycin & 50 & 32.9 & 38.8 & 0.03 \\
\hline Imipenem & 0 & 0 & 0 & - \\
\hline
\end{tabular}

${ }^{a}$ p-value $<0.05$ was considered significant statistically. Antibiotic resistance was categorized as susceptible (including susceptible and intermediate susceptible) and resistant groups which was confirmed by E-test.

Table 2. Antibiotic resistance rates (\%) of extended spectrum beta lactamase (ESBL)-positive and ESBL-negative Klebsiella pneumoniae strains

\begin{tabular}{|l|l|l|l|l|}
\hline Antibiotics & ESBL-positive $(\mathrm{n}=48)$ & ESBL-negative $(\mathrm{n}=68)$ & Total $(\mathrm{n}=116)$ & $\mathrm{p}^{\mathrm{a}}$ \\
\hline Amikacin & 50 & 17.6 & 31 & $<0.001$ \\
\hline Ampicillin & 93.8 & 66.2 & 77.6 & $<0.001$ \\
\hline Ceftazidime & 37.5 & 22.1 & 28.4 & 0.07 \\
\hline Ceftriaxone & 41.7 & 20.6 & 29.3 & 0.014 \\
\hline Ciprofloxacin & 35.4 & 14.7 & 23.3 & 0.009 \\
\hline Co-Amoxiclav & 31.2 & 26.5 & 28.4 & 0.57 \\
\hline Gentamycin & 47.9 & 26.5 & 35.3 & 0.017 \\
\hline Imipenem & 0 & 0 & 0 & - \\
\hline
\end{tabular}

${ }^{a}$ p-value $<0.05$ was considered significant statistically. Antibiotic resistance was categorized as susceptible (including susceptible and intermediate susceptible) and resistant groups which was confirmed by E-test. 
Table 3. Univariate and multivariate analysis of risk factors for extended spectrum beta lactamase (ESBL) E. coli ${ }^{\text {a }}$ strains

\begin{tabular}{|c|c|c|c|c|c|c|}
\hline \multicolumn{2}{|l|}{ Variables } & \multicolumn{3}{|l|}{ ESBL } & \multicolumn{2}{|l|}{ Logistic regression } \\
\hline & & No, n (\%) & Yes, n (\%) & $\mathrm{p}$ & OR $(95 \% \mathrm{CI})$ & $\mathrm{p}$ \\
\hline \multirow[t]{2}{*}{ Gender } & Male & $41(51.2)$ & $30(55.6)$ & 0.6 & & \\
\hline & Female & $39(48.8)$ & $24(44.4)$ & & & \\
\hline \multicolumn{2}{|l|}{ Age (years) } & $30.3 \pm 22.8$ & $38 \pm 26.2$ & 0.07 & $0.99(0.98-1.01)$ & 0.63 \\
\hline \multirow[t]{6}{*}{ Admission wards } & ICU & $8(10)$ & $12(22.2)$ & 0.3 & & \\
\hline & Pediatric & $24(30)$ & $10(18.5)$ & & & \\
\hline & Internal Medicine & $13(16.2)$ & $6(11.1)$ & & & \\
\hline & Surgery & $13(16.2)$ & $10(18.5)$ & & & \\
\hline & Infectious & $11(13.8)$ & $10(18.5)$ & & & \\
\hline & Gynecology & $11(13.8)$ & $6(11.1)$ & & & \\
\hline \multirow{2}{*}{$\begin{array}{l}\text { Admission } \\
\text { duration (days) }\end{array}$} & $\leq 7$ & $62(77.5)$ & $25(46.3)$ & & - & - \\
\hline & $>7$ & $18(22.5)$ & $29(53.7)$ & $<0.001$ & $0.32(0.13-0.7)$ & 0.011 \\
\hline \multirow[t]{2}{*}{ Diabetes } & No & $68(85)$ & $46(85.2)$ & & & \\
\hline & Yes & $12(15)$ & $8(14.8)$ & 0.98 & & \\
\hline \multirow[t]{2}{*}{ Renal Failure } & No & $80(100)$ & $47(87)$ & & & \\
\hline & Yes & $0(0)$ & $7(13)$ & 0.9 & & \\
\hline \multirow{2}{*}{$\begin{array}{l}\text { Admission } \\
\text { History }\end{array}$} & No & $76(95)$ & $33(61.1)$ & & - & - \\
\hline & Yes & $4(5)$ & $21(38.9)$ & $<0.001$ & $0.3(0.08-1.3)$ & 0.1 \\
\hline \multirow{2}{*}{$\begin{array}{l}\text { Antibiotic } \\
\text { History }\end{array}$} & No & $69(86.2)$ & $19(35.2)$ & & - & - \\
\hline & Yes & $11(13.8)$ & $35(64.8)$ & $<0.001$ & $0.14(0.05-0.4)$ & $<0.001$ \\
\hline \multirow[t]{2}{*}{ Steroid History } & No & $76(95)$ & $51(94.4)$ & & & \\
\hline & Yes & $4(5)$ & $3(5.6)$ & 0.9 & & \\
\hline
\end{tabular}

${ }^{a}$ E.coli, Escherichia coli; CI, confidence interval; OR, odds ratio; ICU, intensive care unit.

Table 4. Univariate and multivariate analysis of risk factors for extended spectrum beta lactamase (ESBL) $K$. pneumoniae $^{\text {a }}$ strains

\begin{tabular}{|c|c|c|c|c|c|c|}
\hline \multirow{2}{*}{\multicolumn{2}{|c|}{ Variables }} & \multicolumn{3}{|l|}{ ESBL } & \multicolumn{2}{|c|}{ Logistic regression } \\
\hline & & No, n (\%) & Yes, n (\%) & $\mathrm{p}$ & OR $(95 \%$ CI $)$ & $\mathrm{P}$ \\
\hline \multirow[t]{2}{*}{ Gender } & Male & $36(52.9)$ & $30(62.5)$ & 0.3 & & \\
\hline & Female & $32(47.1)$ & $18(37.5)$ & & & \\
\hline \multicolumn{2}{|l|}{ Age (years) } & $35.4 \pm 24.1$ & $38.2 \pm 25.6$ & 0.54 & & \\
\hline \multirow[t]{6}{*}{ Admission wards } & ICU & $7(10.3)$ & $12(25)$ & 0.4 & & \\
\hline & Pediatric & $13(19.1)$ & $8(16.7)$ & & & \\
\hline & Internal Medicine & $11(16.2)$ & $5(10.4)$ & & & \\
\hline & Surgery & $10(14.7)$ & $7(14.6)$ & & & \\
\hline & Infectious & $12(17.6)$ & $9(18.8)$ & & & \\
\hline & Gynecology & $15(22.1)$ & $7(14.6)$ & & & \\
\hline \multirow{2}{*}{$\begin{array}{l}\text { Admission duration } \\
\text { (days) }\end{array}$} & $\leq 7$ & $50(73.5)$ & $25(52.1)$ & & - & - \\
\hline & $>7$ & $18(26.5)$ & $23(47.9)$ & 0.019 & $0.5(0.2-1.2)$ & 0.14 \\
\hline \multirow[t]{2}{*}{ Diabetes } & No & $58(85.3)$ & $42(87.5)$ & & & \\
\hline & Yes & $10(14.7)$ & $6(12.5)$ & 0.74 & & \\
\hline \multirow[t]{2}{*}{ Renal Failure } & No & $67(98.5)$ & $47(97.9)$ & & & \\
\hline & Yes & $1(1.5)$ & $1(2.1)$ & 0.8 & & \\
\hline \multirow[t]{2}{*}{ Admission History } & No & $64(94.1)$ & $33(72.9)$ & & - & - \\
\hline & Yes & $4(5.9)$ & $13(27.1)$ & 0.003 & $0.44(0.1-1.7)$ & 0.24 \\
\hline \multirow[t]{2}{*}{ Antibiotic History } & No & $55(80.9)$ & $19(39.6)$ & & - & - \\
\hline & Yes & $13(19.1)$ & $29(60.4)$ & $<0.001$ & $0.23(0.09-0.57)$ & 0.002 \\
\hline \multirow[t]{2}{*}{ Steroid History } & No & $64(94.1)$ & $37(77.1)$ & & - & - \\
\hline & Yes & $4(5.9)$ & $11(22.9)$ & 0.012 & $0.47(0.1-1.8)$ & 0.27 \\
\hline
\end{tabular}

${ }^{a}$ K.pneumoniae, Klebsiella pneumoniae; CI, confidence interval; OR, odds ratio; ICU, intensive care unit. 
The most resistance was to ampicillin, and the least resistance was to imipenem. No resistance was detected to imipenem. Anti-microbial resistance was significantly higher among ESBL positive strains (Tables 1 and 2). A multi-drug resistant ESBL (MDR-ESBL) pattern was indicated in 74 of the isolates (29.6\%). Forty (29.9\%) of E. coli and 34 (29.3\%) of $K$. pneumoniae strains were MDR-ESBL ( $\mathrm{p}=0.93)$. Of the ESBL producing strains, $72.5 \%$ were MDR $(\mathrm{p}<0.001, \mathrm{OR}=5.7, \mathrm{CI}: 3.3-9.9)$. By the logistic regression test, the associated risk factors of ESBL positive E. coli were admission duration exceeding 7 days and antibiotic use in the last month, and the associated risk factor for ESBL positive $K$. pneumoniae was antibiotic use during the past month (Tables 3 and 4).

\section{Discussion}

Gram negative strains, including $E$. coli and $K$. pneumoniae, that contain ESBL enzymes have emerged as a great problem in various parts of the world during recent years (1). These enzymes have been expressed increasingly by some isolates with the propensity to spread. The production of ESBLs disturbs a wide spectrum of antibiotic activity, causing major treatment failures and adverse effects on the prognosis of patients. The emergence of ESBLs has created diagnostic difficulties for many clinical microbiology laboratories (12). Our results indicated that the prevalence of ESBL producing E. coli and K. pneumoniae (ESBL-EK) was $40.3 \%$ and $41.4 \%$, respectively (with an average of $40.8 \%$ ). In an investigation by Bazzaz et al. in Iran, the prevalence of ESBL-EK isolates was $59.2 \%$, which was greater than our results (7). Mehrgan et al. indicated that $77.7 \%$ of $K$. pneumoniae strains among admitted patients were ESBL producers, and that also was greater than ours (13). The minimum prevalence rates of isolates expressing the ESBL phenotype were reported in Japan, Singapore, and Sweden (3-8\%) in comparison with higher prevalence rates in Italy (37\%), Turkey (58\%), and Latin American regions (30-60\%) (8). The detection rates of ESBL producing pathogens were reported as 31.7\% in Kuwait (14) and 41\% in the United Arab Emirates (15). These differences may be due to the fact that the studies were concentrated on hospital-acquired infections, special locations of infection, such as blood and urine, or a variety of policies about antimicrobial prescription in various parts of the world. Moreover, genetic variations may be addressed as another cause. The present investigation regarding ESBL producing bacteria indicated relative susceptibility to third-generation cephalosporins (ceftriaxone and ceftazidime) that was consistent with Shakil et al.'s study (16). All ESBL-EK isolates in our survey were sensitive to imipenem, and that was compatible with Shaikh et al.'s results (17). The authors suggested carbapemens as the drug of choice for treatment of ESBL-EK producers (17). In a study by Aminzadeh in Iran, the resistance rate of ESBL-EK to ciprofloxacin was reported to be more than $30 \%$, and that was congruent with our findings; moreover, the amikacin and gentamycin resistance rates were indicated as $10 \%$ and $33.5 \%$, respectively, and both were less than our results (18). In research conducted by Khanfar et al. on ESBL-EK, high resistance rates to amikacin, gentamycin, amoxicillin-clavulanic acid, and ciprofloxacin were reported, and they were greater than our findings (9).

The resistance level of amoxicillin-clavulanic acid against ESBL-EK in the current study was reported as 38.9\% for E. coli and $31.2 \%$ for K. pneumoniae, which was substantially less than the previous study (80\%) (9). It may suggest that the beta-lactam/beta-lactamase inhibitor combinations may be effective in the treatment of some ESBL producing strains that is useful for the prevention of carbapenem resistance. In an investigation in China from May 2013 to February 2014, 45 carbapenem-resistant Enterobacteriaceae (CRE) isolates were produced in a hospital setting. Most of the patients recovered through the use of some kinds of cephalosporins, beta-lactam/beta-lactamase inhibitor combinations, amikacin, and levofloxacin. The drugs of choice included levofloxacin and amikacin (19). It shows the occurrence of carbapenem resistance in some parts of the world, which demands the implementation of control modalities and limitation of the unnecessary use of antimicrobial agents. In this research, the MDR-ESBL pattern was detected in $29.6 \%$ of all isolates, including $29.9 \%$ of E. coli and $29.3 \%$ of K. pneumoniae strains. Aminzadeh reported MDR-ESBL pattern in $26.8 \%$ of the strains, which included $25.8 \%$ of the E. coli and $30 \%$ of the K. pneumoniae isolates, which were less than our findings (18). Kateregga et al. reported that urine was the most common sample for isolation of ESBL organisms (20). It was not in accordance with our results, which showed that there was no association between ESBL-EK and clinical samples $(P=0.88)$. Furthermore, a high level of resistance $(73.8 \%)$ to ceftazidime was substantially more than our survey (20). Our study indicated that there was no significant association between patients' age and ESBL phenotype, which was in harmony with previous investigation (20), but it was incompatible with Khanfar's report that indicated the age of more than 60 as a risk factor of ESBL-EK (9). Kiratisin conducted an investigation and declared that females had a risk factor for ESBLEK that was incongruent with our findings and Kateregga's findings $(20,21)$. The current study showed that the associated risk factors for ESBL producing $E$. coli included admission duration exceeding 7 days $(p=0.011)$ and antibiotic use in the past month $(\mathrm{p}<0.001)$. Moreover, the associated risk factor for ESBL producing $K$. pneumoniae was antibiotic use during the previous month $(\mathrm{p}=0.002)$. These findings were comparable with Shaikh 
et al.'s survey (17). According to previous reports, anti-microbial use is considered as a serious risk factor of ESBLEK infections, therefore the logical use of antibiotics is prudent. In this research, we found no significant association between admission wards and acquisition of ESBL-EK, which was not in harmony with the findings of Khanfar et al. (9). They indicated that admission in intensive care unit (ICU) was a risk factor for isolation of ESBL organisms because the patients in ICU are more likely to have used invasive devices, such as in-dwelling intravascular and urinary catheters. In an investigation by Seni et al., the most ESBL producing strains occurred in surgical wards, which was inconsistent with our results (22). Prolonged use of catheters, unsuitable antimicrobial therapy, long-term duration of hospitalization, and implementation of surgical drainage and nasogastric tubes were the probable mechanisms of their spread. ESBL-EK strains have been identified as the major cause of infection outbreaks globally and have a great role in the failure of infection control. Therefore, it is judicious to detect ESBLs routinely in hospitals to select appropriate antimicrobial agents (8). Common causes of nosocomial infections are ESBLs, so their early detection is helpful in controlling hospital infections. Recently, large numbers of these organisms have been found to be MDR, so their treatment is a serious problem (8).

The strengths of the study include the evaluation of risk factors for ESBL-EK isolates, which has been performed in a few studies, and the application of then E-test as a confirmatory test for the determination of antibiotic resistance in addition to the disk diffusion test. Some limitations of our study were 1) detection of antimicrobial resistance genes was not performed; 2) the sample size was small; and 3) multicenter evaluation was not conducted. So a larger sample size and multicenter and molecular investigations are suggested in the future.

\section{Conclusions}

This study demonstrated a relatively high prevalence of ESBL producing pathogens, however comparatively acceptable antimicrobial susceptibility was seen among them. Most of the ESBL-EK strains were MDR. Among ESBL producers, the most resistance was to ampicillin and the least was to Imipenem. Admission duration and antibiotic use in the last month were significant associated risk factors of ESBL producing isolates. Restriction of antibiotic use, shortening of hospitalization period, early detection of ESBL producers in the hospital, and periodic evaluation of antimicrobial resistance are imperative to control the spread of these bacteria.

\section{Acknowledgments:}

This manuscript was extracted from a research project. The authors acknowledge Kashan University of Medical Sciences for financial support, grant number 9162. We thank the staff of the Microbiology Laboratory at Kashan Shahid Beheshti Hospital for their valuable cooperation. We also appreciate the assistance provided by the staff members of the pediatric, ICU, surgery, internal medicine, gynecology, and infectious wards at Shahid Beheshti Hospital.

\section{Conflict of Interest:}

There is no conflict of interest to be declared.

\section{Authors' contributions:}

All authors contributed to this project and article equally. All authors read and approved the final manuscript.

\section{References}

1) Pitout JD, Laupland KB. Extended-spectrum beta-lactamase-producing Enterobacteriaceae: an emerging public-health concern. Lancet Infect Dis. 2008; 8(3): 159-66. doi: 10.1016/s1473-3099(08)70041-0, PMID: 18291338.

2) Paterson DL. Resistance in gram-negative bacteria: enterobacteriaceae. Am J Med. 2006; 119(6 Suppl 1): S20-8. doi: 10.1016/j.amjmed.2006.03.013, PMID: 16735147.

3) Bradford PA. Extended-spectrum beta-lactamases in the 21st century: characterization, epidemiology, and detection of this important resistance threat. Clin Microbiol Rev. 2001; 14(4): 933-51. doi: 10.1128/cmr.14.4.933-951.2001, PMID: 11585791, PMCID: PMC89009.

4) Roh KH, Uh Y, Kim JS, Kim HS, Shin DH, Song W. First outbreak of multidrug-resistant Klebsiella pneumoniae producing both SHV-12-type extended-spectrum beta-lactamase and DHA-1-type AmpC betalactamase at a Korean hospital. Yonsei Med J. 2008; 49(1): 53-7. doi: 10.3349/ymj.2008.49.1.53, PMID: 18306469, PMCID: PMC2615262. 
5) Nicolau DP. Carbapenems: a potent class of antibiotics. Expert Opin Pharmacother. 2008; 9(1): 23-37. doi: 10.1517/14656566.9.1.23, PMID: 18076336.

6) Nordmann P, Dortet L, Poirel L. Carbapenem resistance in Enterobacteriaceae: here is the storm! Trends Mol Med. 2012; 18(5): 263-72. doi: 10.1016/j.molmed.2012.03.003, PMID: 22480775.

7) Bazzaz BS, Naderinasab M, Mohamadpoor AH, Farshadzadeh Z, Ahmadi S, Yousefi F. The prevalence of extended-spectrum beta-lactamase-producing Escherichia coli and Klebsiella pneumoniae among clinical isolates from a general hospital in Iran. Acta Microbiol Immunol Hung. 2009; 56(1): 89-99. doi: 10.1556/AMicr.56.2009.1.7, PMID: 19388560.

8) Paterson DL, Bonomo RA. Extended-spectrum beta-lactamases: a clinical update. Clin Microbiol Rev. 2005; 18(4): 657-86. doi: 10.1128/cmr.18.4.657-686.2005, PMID: 16223952, PMCID: PMC1265908.

9) Khanfar HS, Bindayna KM, Senok AC, Botta GA. Extended spectrum beta-lactamases (ESBL) in Escherichia coli and Klebsiella pneumoniae: trends in the hospital and community settings. J Infect Dev Ctries. 2009; 3(4): 295-9. doi: 10.3855/jidc.127, PMID: 19759493.

10) Forbes BA, Sahm DF, Weissfeld AS. Bailey \& Scott's Diagnostic Microbiology. 12th ed. St.Louis: Mosby; 2007.

11) Performance Standards for Antimicrobial Susceptibility Testing. 17th Informational Supplement. 2007. Available from: http://www. microbiolab-bg.com/CLSI.pdf.

12) Sharma M, Pathak S, Srivastava P. Prevalence and antibiogram of Extended Spectrum beta-Lactamase (ESBL) producing Gram negative bacilli and further molecular characterization of ESBL producing Escherichia coli and Klebsiella spp. J Clin Diagn Res. 2013; 7(10): 2173-7. doi: 10.7860/jcdr/2013/6460.3462, PMID: 24298468.

13) Mehrgan H, Rahbar M, Arab-Halvaii Z. High prevalence of extended-spectrum beta-lactamase-producing Klebsiella pneumoniae in a tertiary care hospital in Tehran, Iran. J Infect Dev Ctries. 2010; 4(3): 132-8. doi: 10.3855/jidc.488, PMID: 20351452.

14) Mokaddas EM, Abdulla AA, Shati S, Rotimi VO. The technical aspects and clinical significance of detecting extended-spectrum beta-lactamase-producing Enterobacteriaceae at a tertiary-care hospital in Kuwait. J Chemother. 2008; 20(4): 445-51. doi: 10.1179/joc.2008.20.4.445, PMID: 18676224.

15) Al-Zarouni M, Senok A, Rashid F, Al-Jesmi SM, Panigrahi D. Prevalence and antimicrobial susceptibility pattern of extended-spectrum beta-lactamase-producing Enterobacteriaceae in the United Arab Emirates. Med Princ Pract. 2008; 17(1): 32-6. doi: 10.1159/000109587, PMID: 18059098.

16) Shakil S, Ali SZ, Akram M, Ali SM, Khan AU. Risk factors for extended-spectrum beta-lactamase producing Escherichia coli and Klebsiella pneumoniae acquisition in a neonatal intensive care unit. J Trop Pediatr. 2010; 56(2): 90-6. doi: 10.1093/tropej/fmp060, PMID: 19608665.

17) Shaikh S, Fatima J, Shakil S, Rizvi SM, Kamal MA. Risk factors for acquisition of extended spectrum beta lactamase producing Escherichia coli and Klebsiella pneumoniae in North-Indian hospitals. Saudi J Biol Sci. 2015; 22(1): 37-41. doi: 10.1016/j.sjbs.2014.05.006, PMID: 25561881, PMCID: PMC4281604.

18) Aminzadeh Z, Sadat Kashi M, Sha'bani M. Bacteriuria by extended-spectrum Beta-lactamase-producing Escherichia coli and Klebsiella pneumoniae: isolates in a governmental hospital in South of Tehran, Iran. Iran J Kidney Dis. 2008; 2(4): 197-200, PMID: 19377237.

19) Wang $\mathrm{X}$, Chen $\mathrm{G}, \mathrm{Wu} \mathrm{X}$, Wang $\mathrm{L}$, Cai J, Chan EW, et al. Increased prevalence of carbapenem resistant Enterobacteriaceae in hospital setting due to cross-species transmission of the bla NDM-1 element and clonal spread of progenitor resistant strains. Front Microbiol. 2015; 6: 595. doi: 10.3389/fmicb.2015.00595, PMID: 26136735, PMCID: PMC4468908.

20) Kateregga JN, Kantume R, Atuhaire C, Lubowa MN, Ndukui JG. Phenotypic expression and prevalence of ESBL-producing Enterobacteriaceae in samples collected from patients in various wards of Mulago Hospital, Uganda. BMC Pharmacol Toxicol. 2015; 16:14. doi: 10.1186/s40360-015-0013-1, PMID: 26031914, PMCID: PMC4451872.

21) Kiratisin P, Apisarnthanarak A, Laesripa C, Saifon P. Molecular characterization and epidemiology of extended-spectrum-beta-lactamase-producing Escherichia coli and Klebsiella pneumoniae isolates causing health care-associated infection in Thailand, where the CTX-M family is endemic. Antimicrob Agents Chemother. 2008; 52(8): 2818-24. doi: 10.1128/aac.00171-08. PMID: 18505851, PMCID: PMC2493136.

22) Seni J, Najjuka CF, Kateete DP, Makobore P, Joloba ML, Kajumbula H, et al. Antimicrobial resistance in hospitalized surgical patients: a silently emerging public health concern in Uganda. BMC Res Notes. 2013; 6:298. doi: 10.1186/1756-0500-6-298, PMID: 23890206, PMCID: PMC3729663. 